\title{
Fiber Optic Vibration Sensor Based on the Tilted Fiber Bragg Grating
}

\author{
Jiali An, Tao Liu, and Yongxing Jin \\ Institute of Optoelectronic Technology, China Jiliang University, Xueyuan Street, Xiasha Higher Education Zone, \\ Hangzhou 310018, China
}

Correspondence should be addressed to Jiali An; ajlcdr@163.com

Received 22 May 2013; Revised 17 July 2013; Accepted 30 July 2013

Academic Editor: Xinyong Dong

Copyright (C) 2013 Jiali An et al. This is an open access article distributed under the Creative Commons Attribution License, which permits unrestricted use, distribution, and reproduction in any medium, provided the original work is properly cited.

\begin{abstract}
A temperature-insensitive fiber optic vibration sensor based on the tilted fiber Bragg grating (TFBG) is presented. The sensing head is formed by insertion of a small section of MMF between a single-mode fiber and the TFBG. The reflection light from this tilted fiber Bragg grating includes two parts: the reflected Bragg mode and the cladding modes. The cladding modes were coupled back into the core mode as a function of the multimode fiber. The power of the cladding modes is sensitive to vibration, so the external vibration measurement can be obtained through the cladding mode average output power. Experiment results show that the root mean square (RMS) of the detection error of the average power was $0.01 \mu \mathrm{W}$ within the temperature range from 20 to $70^{\circ} \mathrm{C}$, so it is proved to be temperature independent; its frequency response has been tested to $1 \mathrm{KHz}$.
\end{abstract}

\section{Introduction}

Vibration exists in a variety of engineering structures and mechanical systems, and it is very important and valuable to measure and analyze the vibration [1]. Traditional piezoelectric vibration sensor cannot meet the requirement since the sensor technology develops to a higher level, especially in a very harsh environment. Fiber optical sensor has high research value and extensive application prospects in the field of vibration sensors because of the characteristics of high sensitivity, immunity to electromagnetic interferences, chemical corrosion resistance, compact size, fast response, and so on, so the vibration sensor based on fiber Bragg gratings (FBGs) is attractive $[2,3]$. The tilted fiber Bragg gratings (TFBGs) belong to the short-period gratings family. Their grating planes are blazed by an angle in relation to the longitudinal fiber axis. The TFBG can not only make the counterpropagating guided modes couple, but can also couple the core mode to high-order mode and radiate out. The different wavelengths propagated inside the fiber can couple to the fiber cladding at the different angles by controlling the angle of the grating wave vector and the fiber axis. The TFBG has the advantages of both the fiber Bragg grating (FBG) and the long-period fiber grating (LPG) [47] due to its special structure and nature. The TFBG has unique temperature characteristics and cladding mode which is highly sensitive to the outside environment. Recently, TFBG refractive index and bending characteristics used the sensing get much attention [8-14].

In this paper, a new type of fiber vibration sensors based on a tilted fiber Bragg grating (TFBG) is presented. The sensing head is formed by insertion of a small section of MMF between a single-mode fiber and the TFBG. The MMF is used to couple back the counterpropagating cladding modes of the TFBG into the core [15]. The Fiber Bragg grating uses wavelength demodulation to measure physical quantities, so there is the effect of temperature. The average power of the cladding modes is sensitive to vibration, so the external vibration measurement can be obtained through the average output power of the cladding mode. The sensor device is simple due to the intensity demodulation method. And the temperature has little impact on the average output power of the reflection spectra, so it is proved to be temperature independent. 


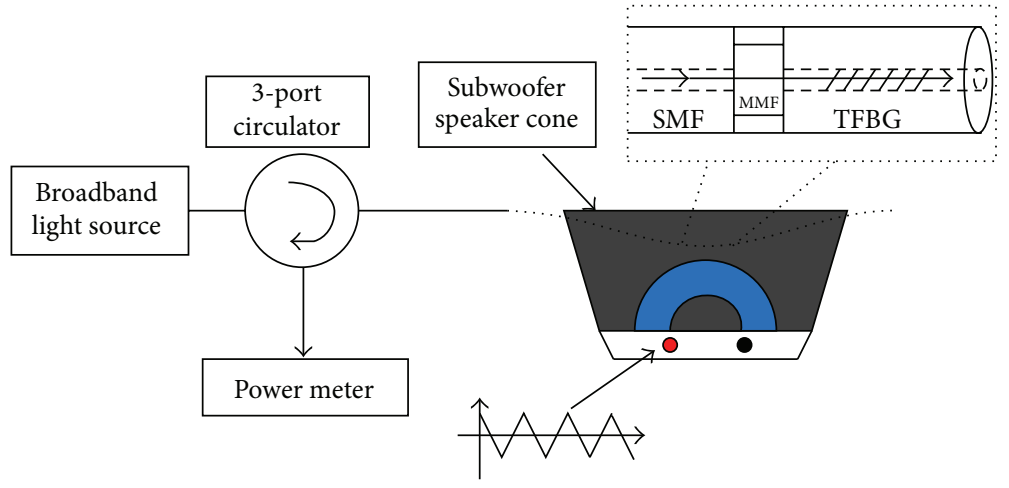

FIGURE 1: Sensing scheme based on the TFBG for vibration measurement. The inset shows the schematic diagram of the sensing head.

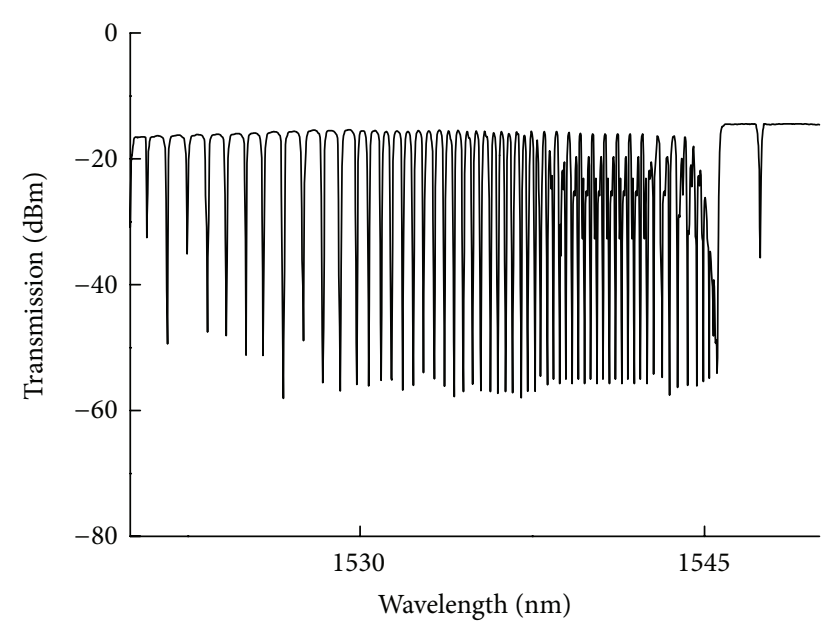

FIGURE 2: Original transmission spectrum of the TFBG.

\section{Sensor Structure and Operation Principle}

The schematic diagram of the vibration sensor is shown in Figure 1. The light from a broadband source was launched into the fabricated sensor through a three-port optical circulator (OC). A $2 \mathrm{~cm}$ long sensor head was glued onto the vibration membrane of the low-frequency speaker. The reflection light from the sensor was measured by using a Newport optical power meter. In the experiment, the sensing head was driven by the speaker vibration membrane with triangular waveform. The schematic diagram of the sensing head is inset in Figure 1. A section of about $2 \mathrm{~mm}-3 \mathrm{~mm}$ multimode fiber is spliced between the SMF and the TFBG, and the MMF used in our experiment has a core diameter of $105 \mu \mathrm{m}$ and clad diameter of $125 \mu \mathrm{m}$. The TFBG was inscribed by UV laser in a hydrogen-loaded germanium-doped SMF by rotating the phase mask at a certain angle in the fiber plane. The length of the TFBG was about $20 \mathrm{~mm}$, and the effective tilted angle is about $5^{\circ}$.

The original transmission spectrum of the TFBG without splicing MMF is shown in Figure 2. The right dip, on the long-wavelength side, corresponds to the Bragg resonance wavelength; the other dips are the result of coupling between

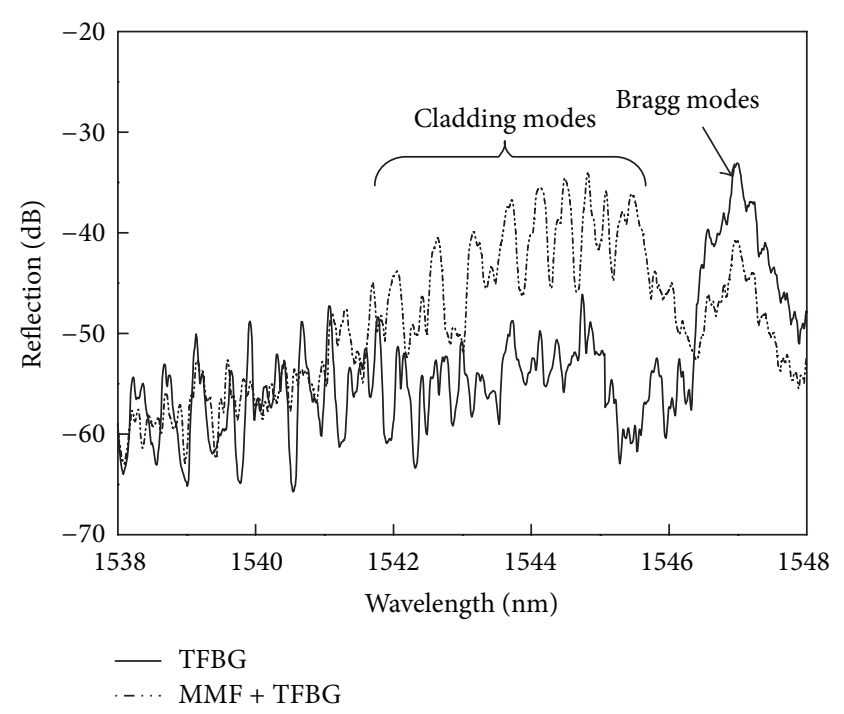

FIGURE 3: Evolution of reflection spectra after being spliced with MMF.

the core mode and the couterpropagating cladding modes of the fiber. The reflection cladding modes emitted from the tilted grating planes cannot propagate for a long distance along the fiber cladding due to the high-index jacket material. The reflection spectra of the proposed sensor compared with those of the bare TFBG are shown in Figure 3. It can be seen that the reflection spectra are different due to the existence of the MMF. For the bare TFBG, the Bragg resonance peak predominated in the reflection spectrum, and the cladding modes are emitted after transmitting for a distance. When the MMF was spliced to the TFBG, there were several cladding modes between $1542 \mathrm{~nm}$ and $1545 \mathrm{~nm}$ emerging in the reflection spectrum. Because the counterpropagating cladding modes were being coupled into the core due to the mode field mismatch between the MMF and the SMF, there were a lot high-order cladding modes of the reflection spectra after splicing MMF. The reflection core mode was decreased by about $5 \mathrm{~dB}-8 \mathrm{~dB}$, as shown in Figure 3, because the mode field mismatch between the SMF and the MMF caused large insertion losses. The length of the MMF segment also affects the recoupling coefficient. Therefore, the optimization of 


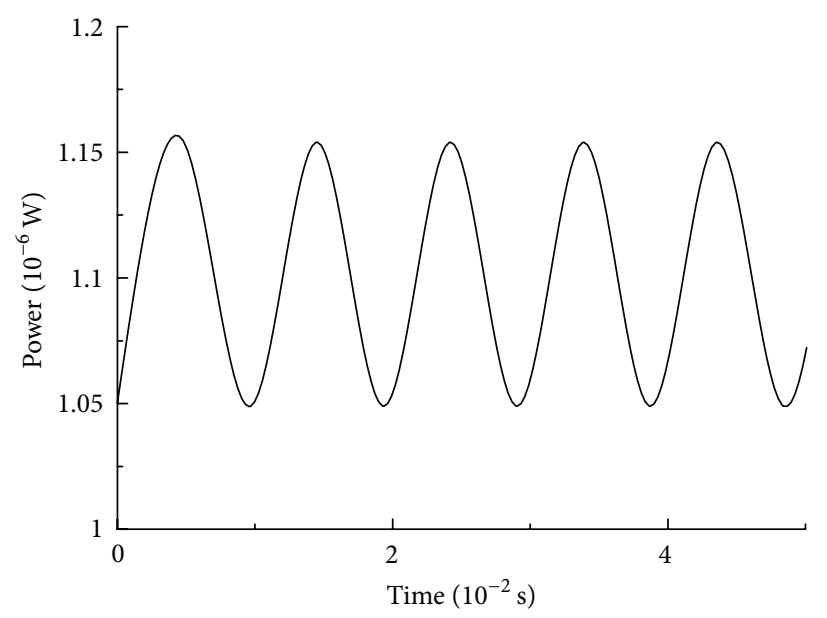

(a) $50 \mathrm{~Hz}$

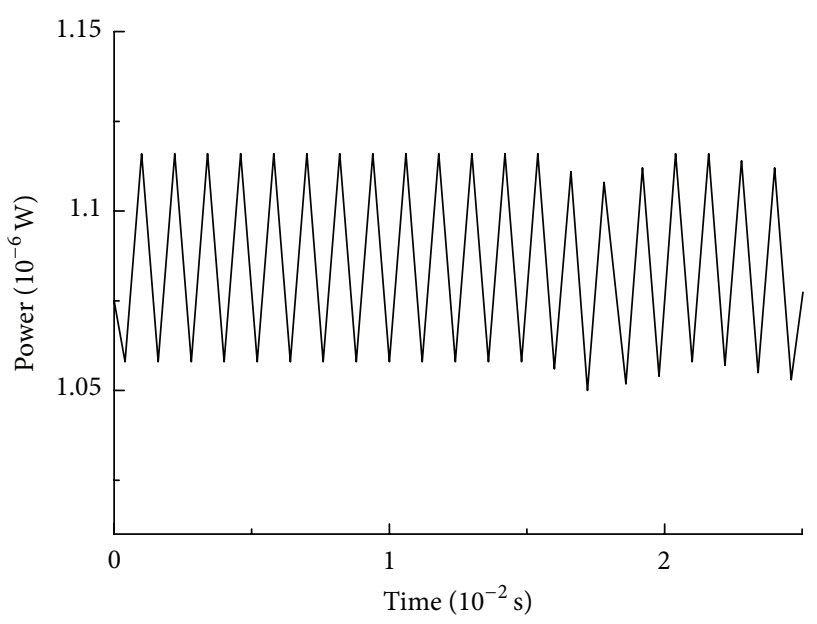

(b) $400 \mathrm{~Hz}$

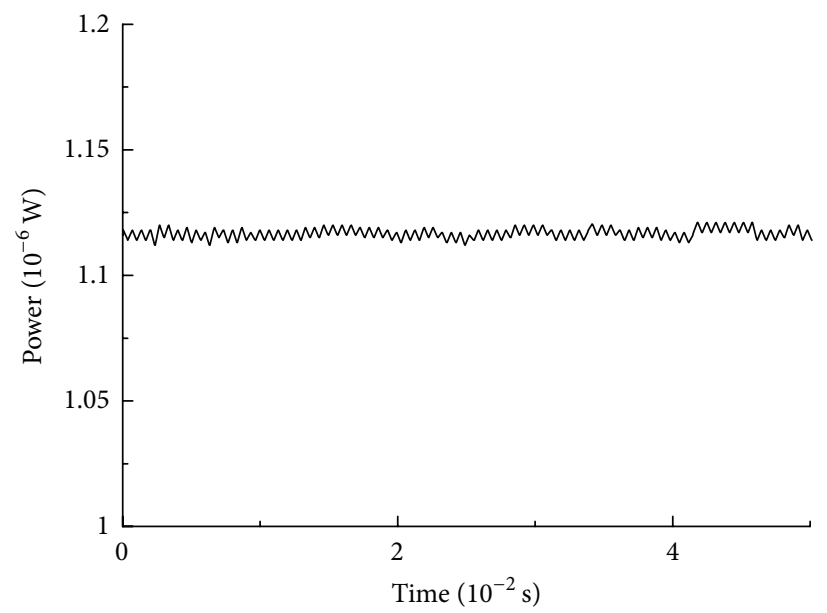

(c) $1 \mathrm{kHz}$

FIGURE 4: Waveform response of the proposed vibration sensor.

the length of the MMF can help to increase the power of the counterpropagating cladding modes, thereby improving the signal intensity of the sensor.

When the external vibration makes the optical fiber sensor bending, the average power of the cladding modes coupled into the core mode changes periodically, and the change cycle corresponds to different vibration frequency. Therefore, the external vibration can be obtained through measuring the reflected average power.

\section{Experimental Results and Discussion}

The sensor attached to the vibration membrane vibrates when the speaker was driven by the signal of triangular wave; thus, the bending of the sensor changes. The average reflection power of the sensor decreases with the increasing of the curvature; therefore, the loss of the cladding modes increase with the increase of the curvature. The lower-order modes are less affected by external influence and can better restrict the energy of mode field. this means that the richer the highorder modes are, the higher sensitive the sensor will be [15].
The reflected power change of the time response of the different frequency measured by the sensor is shown in Figure 4. It is shown that the sensor obtained clear vibration wave signal with a triangular waveform frequency range of $50 \mathrm{~Hz}$ to $400 \mathrm{~Hz}$. The frequency change of the TFBG reflected power was in accordance with the driving frequency. When the driving frequency reach about $500 \mathrm{~Hz}$, the peakpeak value of the frequency response of the reflection power achieves above 0.1 , with an amplitude resolution of $0.06 \mu \mathrm{w} / \mathrm{mm}$. With the increases of frequency, the peak-peak value of the response curve became smaller and smaller until it disappeared. Increasing the vibration frequency, the difference between the vibration frequency and the sensor natural vibration frequency became bigger, so the curvature of the sensor was smaller and smaller and the peak-peak value of the sensor average output power decreased.

The demodulation scheme for the fiber Bragg grating sensor was generally wavelength demodulation, and the wavelength drift corresponds to the external physical changes. However, the reflection cladding mode output power of the TFBG was changed with the external physical quantity. 


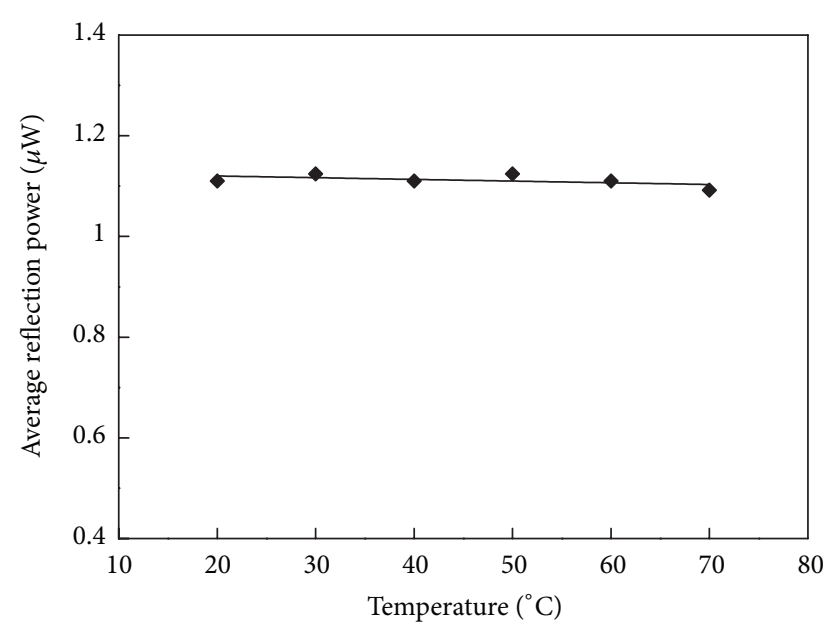

FIGURE 5: Temperature characteristic curve of the proposed vibration sensor.

At the same time, the temperature sensitivity of the TFBG is about $10 \mathrm{pm} /{ }^{\circ} \mathrm{C}$, and the drift of the reflected spectrum is $1 \mathrm{~nm}$ when the temperature changes within $100^{\circ} \mathrm{C}$, so the sensor is proved to be temperature independent. The average output power of the sensor at different temperature is shown in Figure 5. It can be seen that the root mean square (RMS) detection error of the average power was $0.01 \mu \mathrm{W}$ when the temperature ranges from 20 to $70^{\circ} \mathrm{C}$.

The Bragg mode of the TFBG affected the signal-tonoise ratio (SNR) of the vibration signal in the experiment. The average power comprised of the Bragg mode and the cladding mode. The power of the Bragg mode essentially did not change in the vibration measurement process. So, the Bragg mode had no contribution in vibration measurement, it would also affect the measurement sensitivity of the system. The frequency measurement range can further increase if there is an appropriate filter that (such as the long-period grating, and so on) can filter out this power.

\section{Conclusion}

A temperature-insensitive fiber optic vibration sensor based on the TFBG is presented by using the sensitivity characteristic of the cladding mode to external vibration. The sensing head is formed by inserting a small section of MMF between a single-mode fiber and a TFBG. These strongly guided loworder cladding modes can be effectively recoupled to the fiber core via a short section of multimode fiber. The experiment results show that clear vibration wave profile can be obtained when the external vibration frequency changes in the range of 50-400 Hz. In addition, the sensor has the characteristics of the simple structure, demodulation, miniaturization, and so forth, and it has better application prospects in the field of low-frequency of engineering structures and mechanical systems.

\section{References}

[1] R. P. C. Sampaio, N. M. M. Maia, and J. M. M. Silva, "Damage detection using the frequency-response-function curvature method," Journal of Sound and Vibration, vol. 226, no. 5, pp. 1029-1042, 1999.

[2] H. Kang, H. Bang, C. Hong, and C. Kim, "Simultaneous measurement of strain, temperature and vibration frequency using a fibre optic sensor," Measurement Science and Technology, vol. 13, no. 8, pp. 1191-1196, 2002.

[3] H. Somatomo, S. Tanaka, and N. Takahashi, "Vibration sensing of solid using long-period fiber grating," in 19th International Conference on Optical Fibre Sensors, vol. 7004 of Proceedings of SPIE, 70042J, April 2008.

[4] T. Erdogan and J. E. Sipe, “Tilted fiber phase gratings," Journal of the Optical Society of America A, vol. 13, no. 2, pp. 296-313, 1996.

[5] K. S. Lee and T. Erdogan, "Fiber mode coupling in transmissive and reflective tilted fiber gratings," Applied Optics, vol. 39, no. 9, pp. 1394-1404, 2000.

[6] L. Shao and J. Albert, "Compact fiber-optic vector inclinometer," Optics Letters, vol. 35, no. 7, pp. 1034-1036, 2010.

[7] J. Albert, L. Shao, and C. Caucheteur, “Tilted fiber Bragg grating sensors," Laser Photonics Review, vol. 7, pp. 83-108, 2013.

[8] Y. Y. Shevchenko and J. Albert, "Plasmon resonances in goldcoated tilted fiber Bragg gratings," Optics Letters, vol. 32, no. 3, pp. 211-213, 2007.

[9] D. Paladino, A. Cusano, P. Pilla, S. Campopiano, C. Caucheteur, and P. Mégret, "Spectral behavior in nano-coated tilted fiber Bragg gratings: effect of thickness and external refractive index," IEEE Photonics Technology Letters, vol. 19, no. 24, pp. 2051-2053, 2007.

[10] G. Laffon and P. Ferdinand, "Tilted short-period fibre-Bragggrating-induced coupling to cladding modes for accurate refractometry," Measurement Science and Technology, vol. 12, no. 7, pp. 765-770, 2001.

[11] T. Guo, H. Tam, P. A. Krug, and J. Albert, "Reflective tilted fiber bragg grating refractometer based on strong cladding to core recoupling," Optics Express, vol. 17, no. 7, pp. 5736-5742, 2009.

[12] L. Shao, L. Xiong, C. Chen, A. Laronche, and J. Albert, "Directional bend sensor based on re-grown tilted fiber bragg grating," Journal of Lightwave Technology, vol. 28, no. 18, Article ID 5545368, pp. 2681-2687, 2010.

[13] T. Li, X. Dong, C. C. Chan, C. Zhao, and S. Jin, "Powerreferenced optical fiber refractometer based on a hybrid fiber grating," IEEE Photonics Technology Letters, vol. 23, no. 22, pp. 1706-1708, 2011.

[14] X. Dong, Y. Liu, L. Shao, J. Kang, and C. Zhao, “Temperatureindependent fiber bending sensor based on a superimposed grating," IEEE Sensors Journal, vol. 11, no. 11, pp. 3019-3022, 2011.

[15] Y. X. Jin, C. C. Chan, X. Y. Dong, and Y. F. Zhang, “Temperatureindependent bending sensor with tilted fiber Bragg grating interacting with multimode fiber," Optics Communications, vol. 282, no. 19, pp. 3905-3907, 2009. 

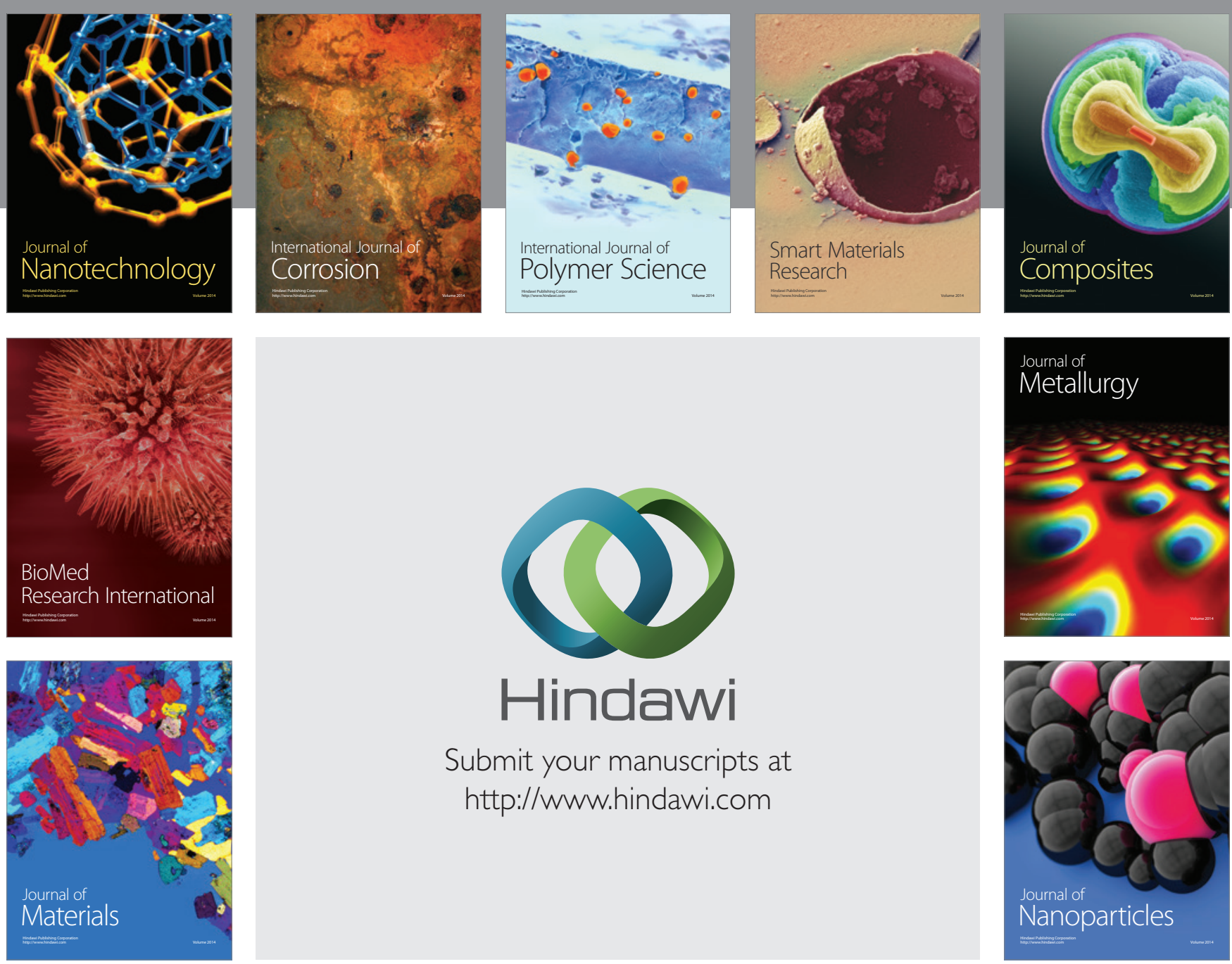

Submit your manuscripts at http://www.hindawi.com
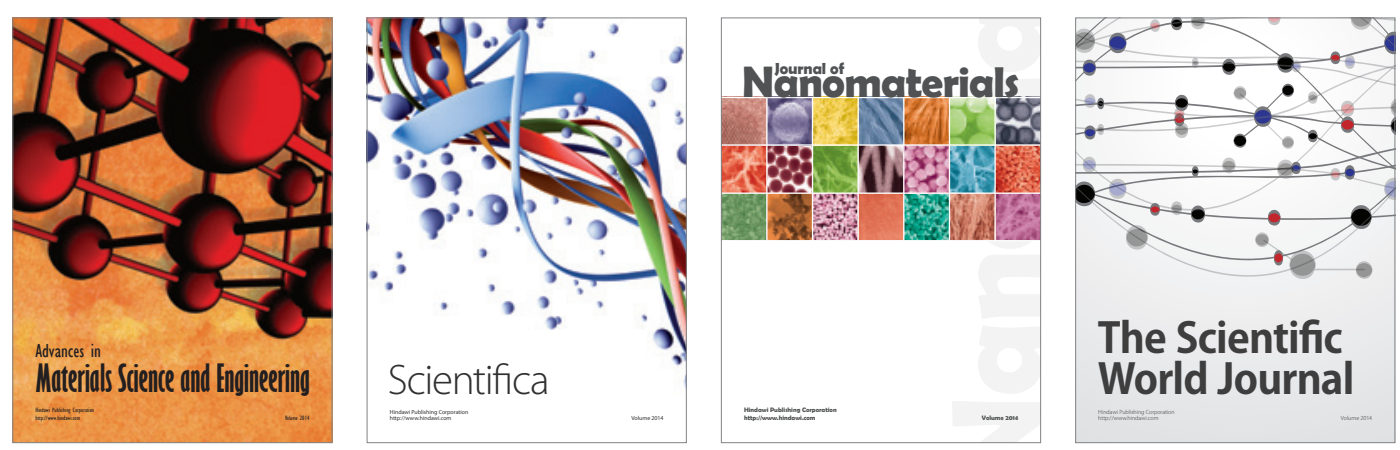

\section{The Scientific World Journal}
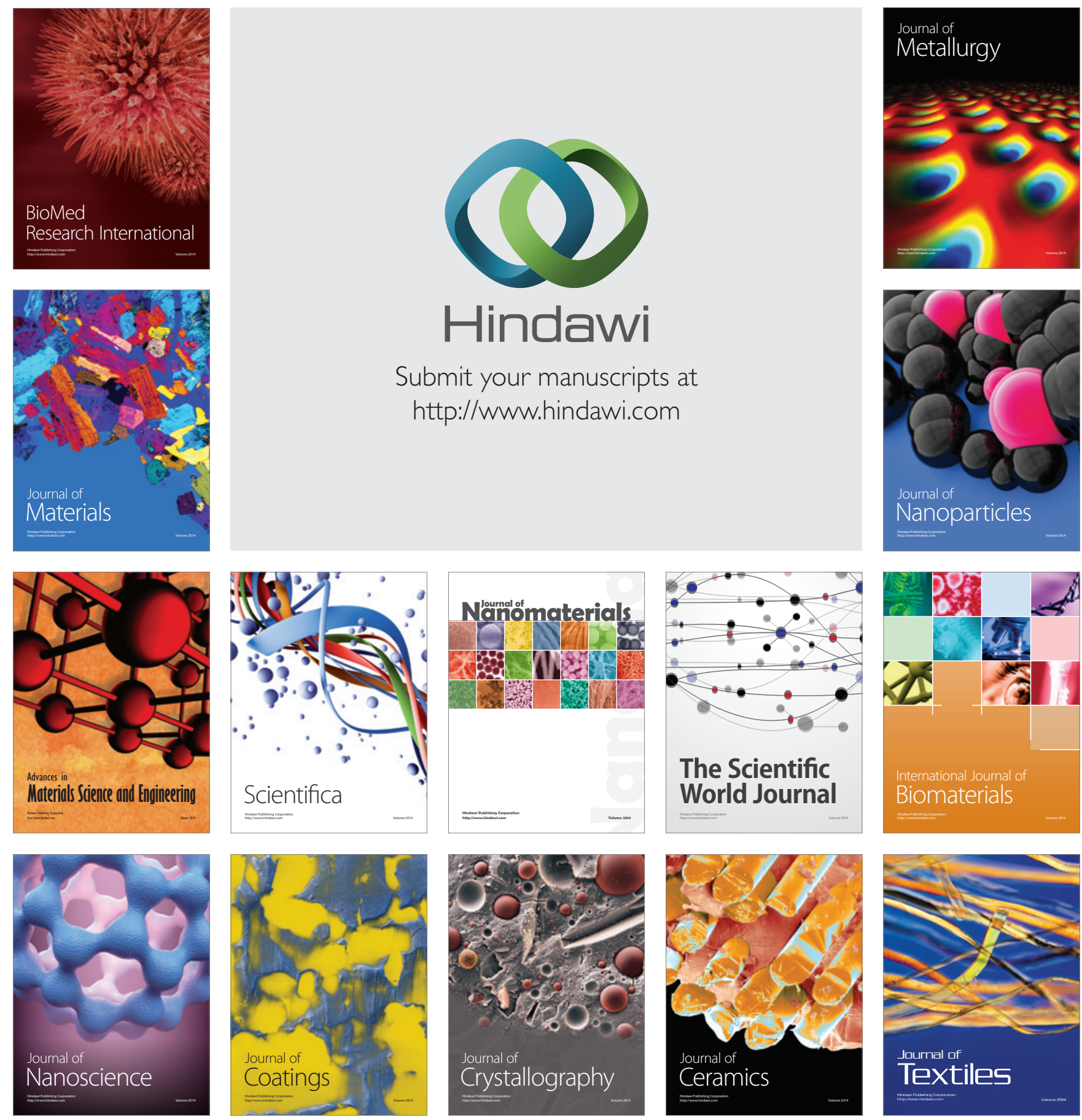